\title{
Immunohistochemical Analysis of Laryngeal Chondrosarcoma: A Case Report

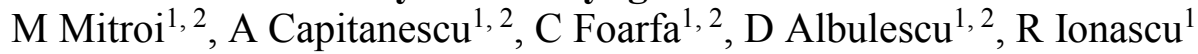

\begin{abstract}
Laryngeal chondrosarcoma is a rare entity, reportedly comprising less than $1 \%$ of all laryngeal tumors. Obtaining the necessary tissue for a histological examination can be difficult. Another difficult step is distinguishing a chondrosarcoma from a chondroma. This differentiation can be achieved through immunohistochemical analysis.
\end{abstract}

We present the histopathological and immunohistochemical aspects of a laryngeal chondrosarcoma, well differentiated and with low grade malignancy, located on the cricoid cartilage. Anatomopathological examination confirms the diagnosis of low-grade malignancy chondrosarcoma and CT scans show no metastasis, therefore the tumor is removed through total laryngectomy with primary pharyngeal reconstruction with a favourable evolution.

The histopathological examination used the classical Hematoxylin-Eosin technique and the immunohistochemical method was based on soluble immunoenzymatic complexes.

This is the first article in the literature that analyzes the immunohistochemical aspects of the laryngeal chondrosarcoma. We consider this study very important beacause of the high difficulty level of obtaining the necessary bioptic material and differentiating it from a laryngeal chondroma. The four antibodies we studied allow us to give the certain diagnosis of a laryngeal chondrosarcoma.

Keywords: Chondrosarcoma, larynx, immunohistochemical analysis, vimentine, $\alpha$-actin

From: ${ }^{1}$ Craiova Clinical and Emergency County Hospital and ${ }^{2}$ University of Medicine and Pharmacy, Craiova, Romania.

Correspondence: Dr M Mitroi, ENT Department, University of Medicine and Pharmacy, Str. Petru Rares, Nr. 2, 200349, Craiova, Romania. E-mail: mhlmitroi@yahoo.com 


\section{INTRODUCTION}

Non-epidermoid cancers of the larynx like chondrosarcoma are an uncommon form of neoplasm, accounting for less than $1 \%$ of cancers of the larynx (1), and about $0.1 \%$ of all neoplasms of thehead and neck region (2). The etiology of the disease is unknown, although it is usually assumed to derive from disordered ossification of the laryngeal cartilage (3-5).

We present the clinical and immunohistochemical analysis of a laryngeal chondrosarcoma, located on the cricoid cartilage, well differentiated and with low grade malignancy.

\section{CASE REPORT}

A 66 year-old male patient presented to our ENT Department with one year history of increasing dyspnea, stridor and weight loss. Indirect laringoscopy revealed a subglottic tumoral mass, obstructing $80 \%$ of its circumference. We performed urgent tracheotomy, under local anesthesia. The direct microlaryngoscopy that we performed afterwards emphasizes a firm yellow-white subglottic tumor mass, located on the posterior wall of the cricoid. We collected biopsy from the tumor and we performed a histopathological and immunohistochemical examination.

After establishing the anatomopathological diagnosis of low-grade malignancy chondrosarcoma, we searched for methastatic lesions using CT scan, without finding any evidence of metastasis. We performed a total laryngectomy with primary pharyngeal reconstruction due to tumoral extension below the lower edge of the cricoid cartilage. Postoperative evolution was favorable, without any complications or relapse. 
The entire larynx was delivered to the Anatomopathological Department of the hospital for histological and immunohistochemical analysis. Macroscopic appearance - it was a unique whitish polypoid, sessile tumor, with variable consistency, gelatinous areas, in the long axis diameter of $3 \mathrm{~cm}$, which is located on the subglottis, under the right vocal cord.

The tissues were prepared using standard technique of paraffin inclusion through the following stages: fixation in $10 \%$ buffered formalin, washing in $80 \%$ alcohol, dehydration by successive alcohol baths, clarification and paraffin embedding. For staining we used the classic Hematoxylin- Eosin (HE) technique.

The immunohistochemical method was based on soluble immunoenzymatic complexes LSAB/HRP (Labeled Streptavidin Biotin) method and the following antibodies: S100, alphasmooth muscle actin, vimentin, Ki-67.

Table 1: Antibodies used in this study

\begin{tabular}{lllll}
\hline Antibody & Source & Clone & Dilution & Antigen retrieval \\
\hline $\begin{array}{l}\text { S100 } \\
\begin{array}{l}\text { Alpha-smooth } \\
\text { muscle actin }\end{array}\end{array}$ & DAKO & Policlonal & $1: 500$ & - \\
$\begin{array}{l}\text { Vimentin } \\
\text { Ki-67 }\end{array}$ & 1A4 & $1: 50$ & 3 cycles citrate buffer \\
& THERMO & V9 & $1: 100$ & 5 cycles citrate buffer \\
\hline
\end{tabular}

\section{DISCUSSION}

The first chondrosarcoma of the larynx was described by New in 1935 (6). Damiani estimated that there are 600 cases in the literature (7). A systematic review published by Chin in 2016 (8) mentions 592 cases in specialty literature. In 178 of the cases the surgical approach was local 
excision and in 174 cases total laryngectomy was used (an approximately equal proportion). In our case, the patient was a 66 year-old male, meeting the criteria established in the literature.

Histological diagnosis of laryngeal chondrosarcoma is based on Lichtenstein and Jaffé's criteria for diagnosis of malignant cartilaginous tumors described in 1943 (9). In 1977, Evans grouped chondrosarcomas in grades I, II and III based on mitotic rate, cellularity and nuclear size (10). Grade I chondrosarcoma is histologically characterized by the exclusive presence or marked preponderance of small, densely-straining nuclei and an intercellular background that varies from chondroid to myxoid. Grade II chondrosarcomas contain areas of nuclei in a significant proportion with a low mitotic rate. Grade III chondrosarcoma is characterized by the presence of two or more mitoses (10).

From a microscopic point of view, laryngeal chondrosarcoma is a malignant mesenchymal tumor. The histological types of chondrosarcoma can be: conventional, with clear cells and dedifferentiated chondrosarcoma (combination of high grade chondrosarcoma and noncartilaginous sarcoma). In our case, it was the conventional type chondrosarcoma, with low-grade malignancy (grade I chondrosarcoma), characterized by low cellularity and consisting of large chondrocytes, uneven hyperchrome nucleus with irregular outline, some incised, others binucleolated (Fig. 1).

Necrosis and atypical mitosis were absent. Recent findings indicate that chondroma and chondrosarcoma of the larynx are closely related, either synchronously (areas of both tumor types within the same lesion) or metachronously (malignant transformation of chondroma over time) (11). It is important to perform an immunohistochemical analysis, since it is the only one able to distinguish between the two tumors. The IHC profile for conventional chondrosarcoma is characterized by diffuse positivity for Vimentin and S100 expression, low Ki-67 proliferation 
index, and an $\alpha$-actin which can be positive in rare tumor cells (12). S100 - It is a low molecular weight protein and cytoplasmatic marker for histiocytes, chondrocytes, lipocytes, striated muscle, Schwann cells and also glial and myoepithelial cells. In our case, the marker was positive, having a diffuse disposal in tumoral chondrocytes (Fig. 2). Smooth muscle actin ( $\alpha$-actin) - It is a cytoplasmatic marker for smooth muscle cells, myofibroblasts, myoepithelial and endothelial cells. In our case, it was intensely positive in isolated tumor cells (Fig. 3).

Vimentin - It is a mesenchymal cytoplasmatic marker for fibroblasts, muscle cells, chondroblasts, osteoblasts, endothelial cells, hematopoietic cells. In our case, the tumor cells had an intense and diffuse response for vimentin (Fig. 4). Ki-67 - It is a nuclear marker expressed in phases G1, S, G2 and M of the cell cycle. In our case, Ki-67 was rarely positive on tumor cells ( $\leq$ 2\%) (Fig. 5). The recommended treatment of laryngeal chondrosarcomas is conservative surgical excision, if possible $(12,13)$. Total laryngectomy is recommended when laryngeal chondrosarcoma develops from cricoid cartilage, making the preservation of larynx functions impossible (14) and in cases of relapse (15). In our case, we opted for total laryngectomy.

The five-year survival rate and tendency of metastasis depends on the differantion grade of the tumor. Low grade chondrosarcoma has a 90\% five-year survival rate and shows no tendency of metastasis (16). In our case, the patient is still alive four years after his surgical intervention. 


\section{REFERENCES}

1. Soumya Ghatak, Tamoghna Jana (2008). Chondrosarcoma of the arytenoids - a rare laryngeal malignancy. Indian J. Otolaryngol. Head neck Surg (October-december 2008) 60: $376-8$.

2. Coca-Pelaz A, Rodrigo JP, Triantafyllou A, et al. Chondrosarcomas of the head and neck. Eur Arch Otorhinolaryngol 2014; 271: 2601-9.

3. Brandwein $\mathrm{M}$, Moore S, Som P, Biller H. Laryngeal chondrosarcomas: a clinicopathologic study of 11 cases, including two "dedifferentiated" chondrosarcomas. Laryngoscope 1992; 102: 858-67.

4. Casiraghi O, Martinez-Madrigal F, PinedaDaboin K, Mamelle G, Resta L, Luna MA. Chondroid tumors of the larynx: a clinicopathologic study of 19 cases, including two dedifferentiated chondrosarcomas. Ann DiagnPathol 2004; 8: 189-97.

5. Righi S, Boffano P, Pateras D, et al. Chondrosarcoma of the laryngeal thyroid cartilage. J Craniofac Surg 2015; 26: e478-9.

6. New GB. Sarcoma of the larynx: report of two cases. Arch Otolaryngol 1935; 21: 64852.

7. Damiani V, Crosetti E, Rizzotto G, et al. Well and intermediatedifferentiated laryngeal chondrosarcoma: toward conservative surgery? Eur Arch Otorhinolaryngol 2014; 271: 3 37-44.

8. Chin OY, Dubal PM, Sheikh AB, Unsal AA, Park RC, Baredes S, Eloy JA. Laryngeal chondrosarcoma: A systematic review of 592 cases. Laryngoscope 2016; doi: 10.1002/lary.26068.

9. Lichtenstein L, Jaffe HL. Chondrosarcoma of bone. Am J Pathol 1943; 19: 553-89. 
10. Evans HL, Ayala AG, Romsdahl MM. Prognostic factors in chondrosarcomaof bone: a clinicopathologic analysis with emphasis on histologic grading. Cancer 1977; 40: 818-31.

11. Baatenburg de Jong RJ, van Lent S, Hogendoorn PC. Chondroma and chondrosarcoma of the larynx. Curr Opin Otolaryngol Head Neck Surg 2004; 12: 98-105.

12. Bruce M Wenig, Clara S Heffess. Atlas ofhead and neck pathology. Second edition, Saunders Elsevier, pag 512-4, 2008.

13. Thompson LD, Gannon FH. Chondrosarcoma of the larynx: a clinicopathologic study of 111 cases with a review of the literature. Am J SurgPathol 2002; 26: 836-51.

14. Oliveira JF, Branquinho FA, Monteiro AR, Portugal ME, Guimarães AM. Laryngeal chondrosarcoma-ten years of experience. Braz J Otorhinolaryngol 2014; 80: 354-8.

15. Sauter A, Bersch C, Lambert KL, Hörmann K, Naim R. Chondrosarcoma of the larynx and review of the literature. Anticancer Res 2007; 27: 2925-9.

16. Zhou HW, Wang J, Liu Y, Zhang HM. Recurrent chondrosarcoma of the larynx: A case report and literature review. Med 2016; 95: e4118. doi: 10.1097/MD.0000000000004118. 


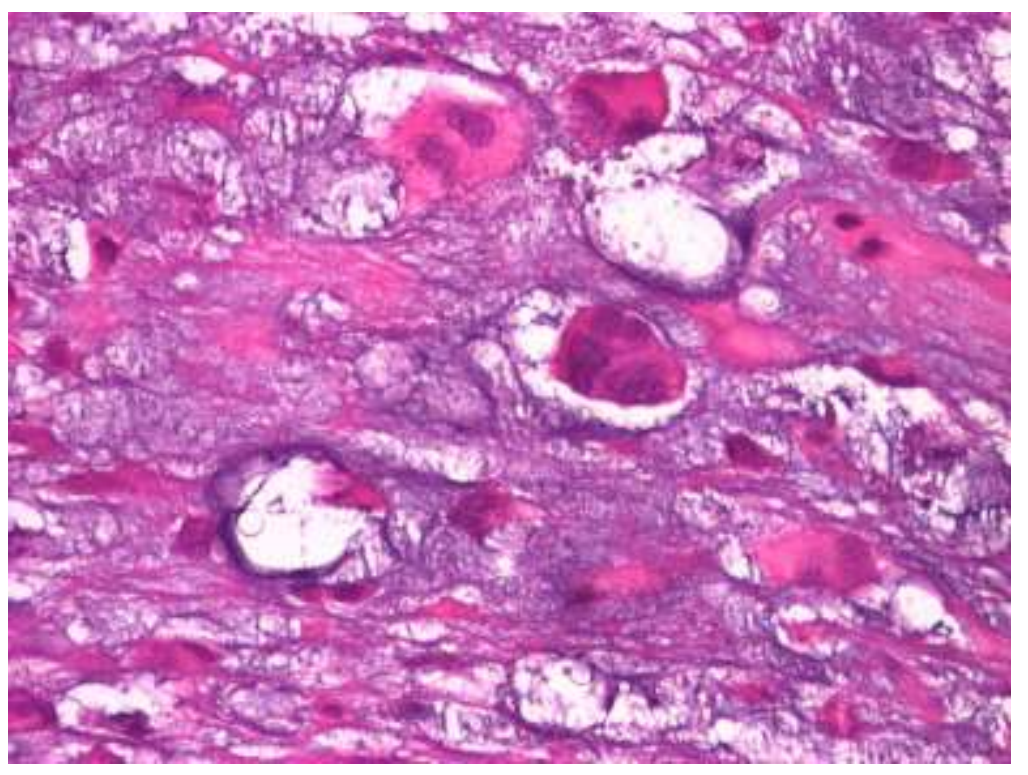

Fig. 1: Grade I chondrosarcoma - Chondrocytes with hypertrophic and irregular nuclei, binucleated. HE, 200x.

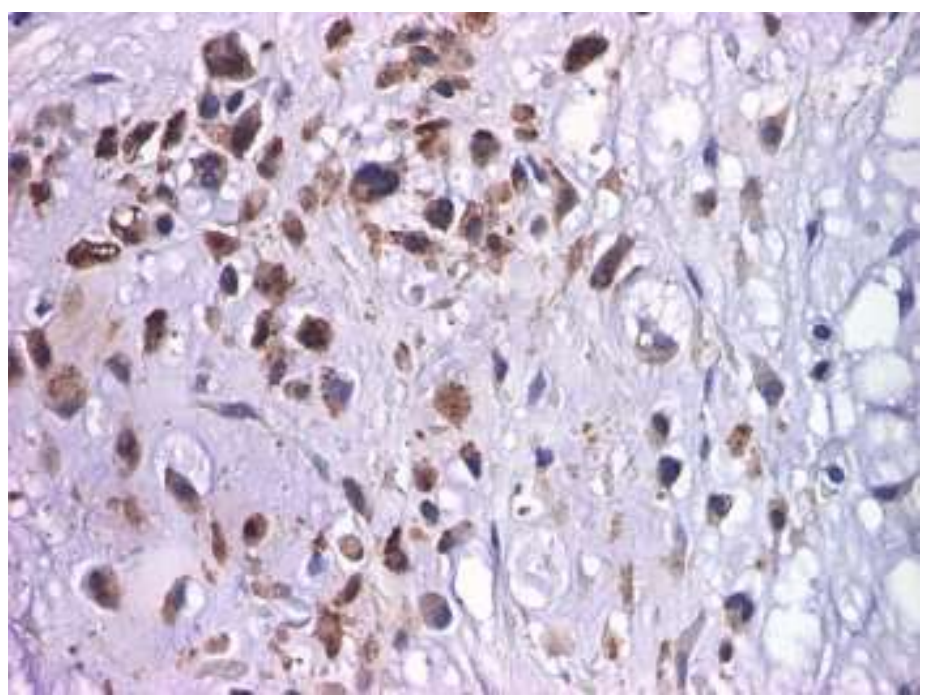

Fig. 2: Grade I chondrosarcoma - S100 intense positive, with diffuse disposal on tumor cells. IHC, 200x. 


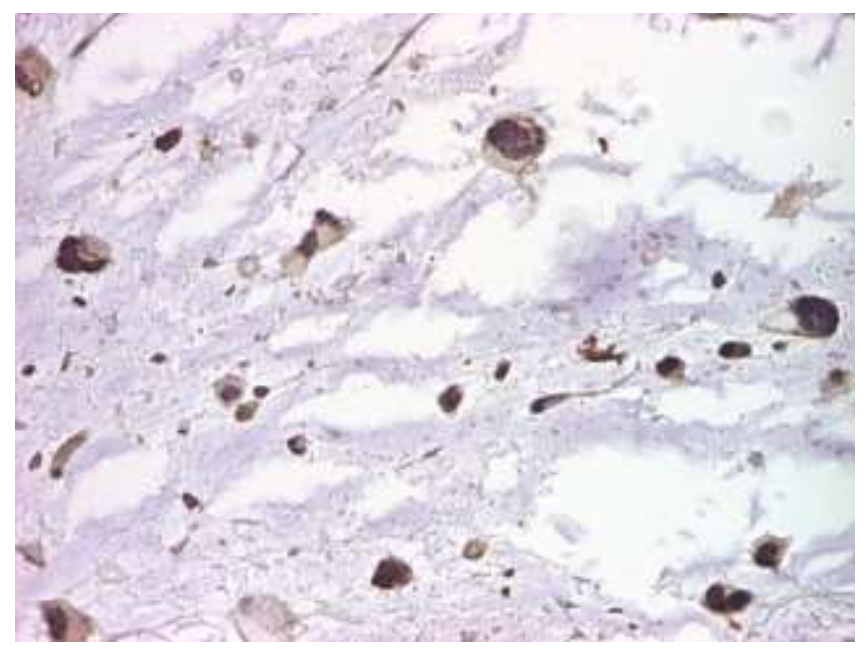

Fig. 3: Grade I chondrosarcoma $-\alpha$-actin intensely positive on isolated tumor cells. IHC, 200x.

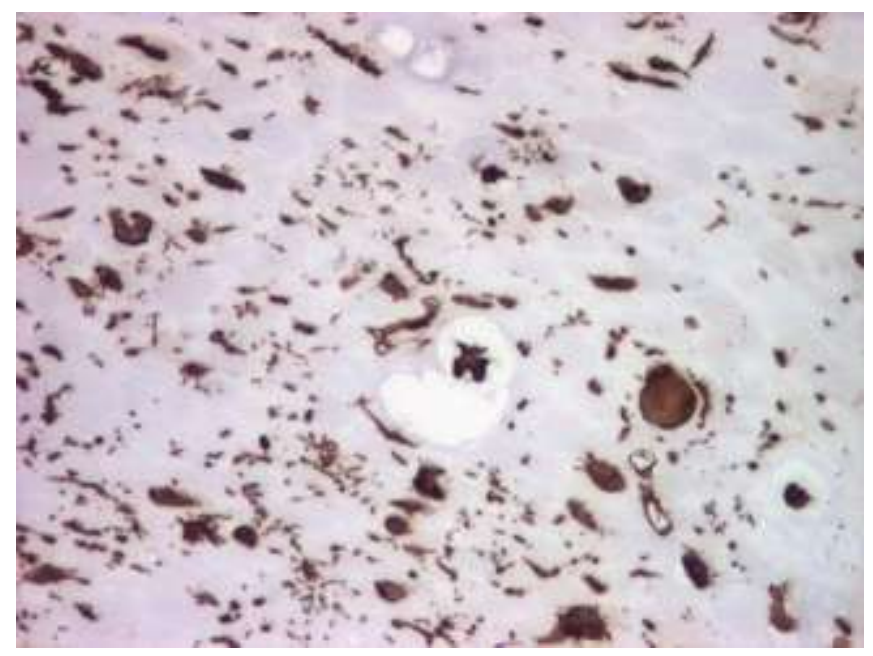

Fig. 4: Grade I chondrosarcoma - Intense and diffuse response for vimentin on tumor cells. IHC, 100x. 


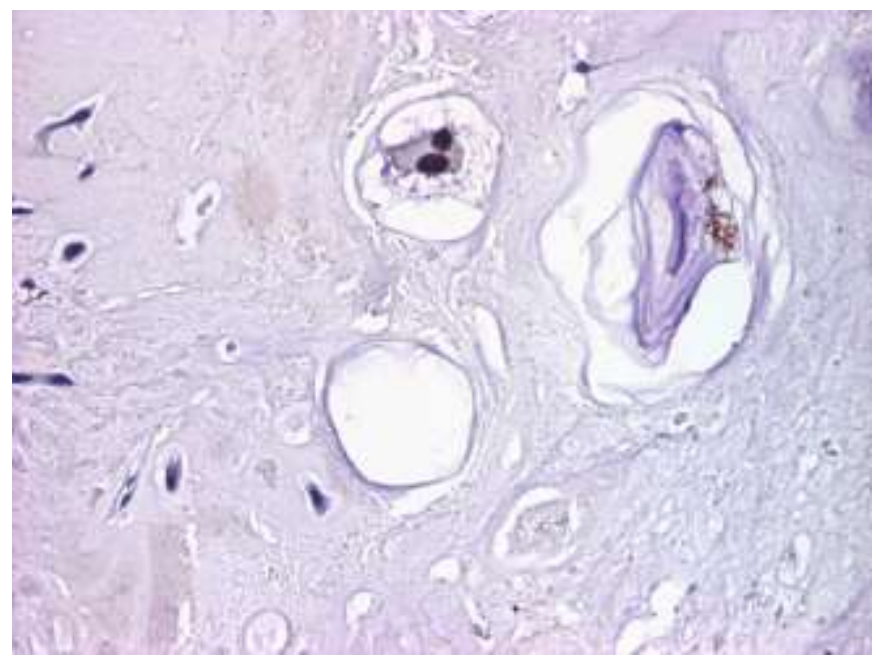

Fig. 5: Grade I chondrosarcoma - Ki-67 intense positive on isolated tumor cells. IHC, 200x. 